\title{
THE ROLE OF EHEALTH IN DISASTERS: A STRATEGY FOR EDUCATION, TRAINING AND INTEGRATION IN DISASTER MEDICINE
}

\author{
Anthony C Norris PhD ${ }^{1}$, Jose J Gonzalez PhD ${ }^{2}$, David T Parry PhD ${ }^{1}$, Richard E Scott PhD ${ }^{3}$, Julie Dugdale \\ PhD $^{4}$, Deepak Khazanchi PhD 5 \\ ${ }^{1}$ School of Computer and Mathematical Sciences, AUT University, New Zealand \\ ${ }^{2}$ Center for Integrated Emergency Management, University of Agder, Norway \\ ${ }^{3}$ Department of Community Health \& Family Medicine, University of Calgary \& NT eHealth Consulting, Canada \\ ${ }^{4}$ Laboratoire d'Informatique de Grenoble, University of Alps, France \& University of Agder, Norway \\ ${ }^{5}$ College of Information Systems \& Technology, University of Nebraska, Omaha, USA
}

\begin{abstract}
This paper describes the origins and progress of an international project to advance disaster eHealth (DEH) - the application of eHealth technologies to enhance the delivery of healthcare in disasters. The study to date has focused on two major themes; the role of DEH in facilitating inter-agency communication in disaster situations, and the fundamental need to promote awareness of DEH in the education of disaster managers and health professionals. The paper deals mainly with on-going research on the second of these themes, surveying the current provision of disaster medicine education, the design considerations for a DEH programme for health professionals, the key curriculum topics, and the optimal delivery mode.
\end{abstract}

Keywords: disaster eHealth; disaster medicine; disaster management; eHealth; curriculum

\section{Introduction}

A survey of recent disasters, whether they are natural catastrophes, human technological errors, epidemics, wars, or terrorist activities, reveals their destructive impact on infrastructure and economies, and the grim costs of individual lives and misery. ${ }^{1,2}$ A perceived increase in the incidence of these events has motivated the development of disaster management (DMAN) as a methodical discipline to respond to and mitigate their often terrible effects. Whilst the media predictably focus on the immediate aftermath of a catastrophe, DMAN tackles issues at all stages of the disaster cycle; mitigation, preparedness, response, and recovery. ${ }^{3}$
In a major disaster there are invariably human casualties who need rapid treatment for trauma and the specialised field of disaster medicine (DMED) ${ }^{4}$ has evolved for this purpose. DMED applies public health and specialist tools to define clinical protocols in a disaster, and to teach the competencies required by clinical personnel. ${ }^{5}$ The health consequences of such events, initially exhibited as fatalities and injuries, are frequently encountered years later as chronic and mental illnesses. ${ }^{6}$

Whilst DMAN is beginning to deploy information and communication technologies (ICTs) to good effect, ${ }^{7-8}$ there is little systematic use of eHealth tools in DMED., ${ }^{9,10}$ These technologies, such as the electronic health record (EHR), telehealth, decision support systems, data analytics, RFID, Internet of Things, cloud computing, and social media etc, are revolutionising the planning and delivery of mainstream healthcare. ${ }^{11}$ Their ability to ensure health information and health-related services are available anytime and anywhere, to automate workflows, and provide seamless care for immediate intervention or longer-term treatment, make these technologies extremely relevant to the needs of disaster healthcare They also 'democratise' healthcare by encouraging the active participation of the consumer in the care process, an important asset in a situation where professional care may be in short supply.

Crucially, however, DMED clinicians are seldom trained to be aware of these technologies, let alone to acquire competency in their use.

These considerations have led to an international project to develop a new paradigm of disaster 
eHealth - the application of eHealth technologies to assist the prognosis and treatment of the sick and injured in a disaster and to support appropriate care in the post-disaster situation. Disaster eHealth (DEH) can be thought of as a domain at the intersection of three constituent fields; disaster management, disaster medicine, and eHealth.

This paper outlines the DEH project and the progress made to date as a prelude to describing the development of a strategy for a key issue that has emerged from the study - the fundamental need for education and training on the role and use of eHealth technologies in disasters.

\section{The DEH Project and Progress to Date}

The DeH project began in 2014 through an initial collaboration between Auckland University of Technology (AUT) in New Zealand and the University of Agder (UiA) in Norway. ${ }^{12}$ Both universities have specialisms in disaster management and eHealth. The consortium was joined in 2015 by the University of Nebraska, Omaha in the USA bringing disaster medicine and related expertise to the partnership. It has subsequently grown further to embrace researchers from universities in Canada, Bulgaria, Sweden, and France.

Foundation work has involved a rigorous study reviewing the published literature on the three component fields of DEH to determine its scope, and the opportunities and limitations attending its application. ${ }^{12}$ The results from over 1,200 peerreviewed publications are being analysed to identify which eHealth technologies are currently most prevalent in disaster healthcare, what they are used for, and in which phases of the disaster cycle they occur. The findings will be used to develop use cases for DEH applications and to identify gaps where eHealth technologies are not yet applied but could bring benefit. These data will allow the construction of a range of scenarios for a Delphi study to determine the DEH technologies and applications that have the greatest potential in terms of care quality, access, cost control, and, of course, their robustness, ease of use, and integration with existing care processes.

The literature survey and the analysis to date have identified several themes, two of which are of key importance and have led to parallel subprojects. First is the need for meaningful communication between disaster managers and disaster medicine specialists. Disaster situations produce rapidly changing scenarios, incomplete data, limited time to make decisions, and high stress levels. Unsurprisingly, post-event analysis reveals that communication and coordination across these agencies is often lacking, leading to delayed, substandard, inappropriate, or even unavailable care. ${ }^{13}$ Moreover, the DMAN and DMED communities have different origins, training, and priorities so that misunderstandings can arise over terminology and intent leading to conflicting practical, if not aspirational, goals. This concern has prompted Bissell to the comment that, "Emergency management and the health sector are natural allies that have, seemingly, only recently begun to recognize each other". ${ }^{14}$ Although this comment was made in 2005, in many countries, little seems to have changed in the intervening decade to refute this concern.

The communication theme is the subject of a separate paper $^{15}$ and the remainder of the present paper focuses on a concomitant strategy to advance the second theme; the education and training of disaster medicine specialists and associated health personnel in the understanding and practice of disaster eHealth.

\section{Current Provision of Disaster Medicine Education}

A brief review of the current provision of DMED education provides a platform for increasing awareness and competence in DEH.

The appalling 9/11 terrorism attacks in New York in 2001 gave the impetus to boost DMED education and training. In 2003, the Association of American Medical Colleges declared an understanding of disaster medicine as vital to a successful response during mass-casualty (especially bioterrorism) events and recommended it as an integral part of the undergraduate medical curriculum. ${ }^{16}$ In the same year, the World Association for Disaster and Emergency Medicine (WADEM) began the development of "International Standards and Guidelines on Education and Training for Disaster Medicine". ${ }^{17,18}$ Rather than prescribe a single curriculum monitored by a professional body, the aim was to provide a framework for organisations to promote their own multi-disciplinary, evidence-based, education for all 
members of the healthcare community.

Universities, mainly in the USA and Europe have responded to the WADEM initiative by developing local, ${ }^{19}$ national, ${ }^{20}$ and even international, ${ }^{21}$ programmes in DMED. However, DMED experts continue to bemoan the low provision of DMED education that leads to a situation where health personnel lack even a basic knowledge of disaster medicine. ${ }^{22-24}$

With limited coverage of even the basic principles and practice of DMED it is easy to see why DEH receives little attention in undergraduate DMED curricula.

The limited provision of undergraduate DMED education can perhaps be explained by the constraints on already over-full undergraduate curricula. ${ }^{25}$ There are more opportunities at the postgraduate level where programmes can be tailored to the needs of advanced professionals. Thus, the European Masters Degree in Disaster Medicine has been offered to around 400 health practitioners since it began in $2000 .^{21} \mathrm{~A}$ comprehensive review of education and training programmes for crisis management in the European Union collates more than 30 DMED-related postgraduate programmes targeted at doctors, nurses, and other health professionals, and extending the early focus on bioterrorism to a fullrange of disaster scenarios. ${ }^{26}$

Even so, most postgraduate programmes in DMED are, like their undergraduate precursors, full-time, on-site courses with no systematic treatment of DEH. Some universities have begun to offer their courses by distance and blended learning modes ${ }^{27}$ strangely however, the literature records few instances of short, in-service courses in DMED. ${ }^{28,29}$ This bias towards in-depth, full-time programmes that offer degrees is understandable in terms of career progression but it would appear to miss opportunities to reach a wider group of care practitioners who want to upskill in disaster healthcare and response. This group would be better served by short courses that can often be spun off and adapted from longer, degree programmes and customised for the intended audience thereby reducing the development overhead.

\section{Projected Audience, Level, and Type of a Proposed DEH Course}

Although requiring more detailed study, the above observations suggest ways to raise awareness of DEH so that disaster medicine specialists can assess the value of appropriate technologies, develop protocols and competencies for their use, and integrate them with existing practice.

The introduction of DEH principles into the undergraduate curriculum, whilst attractive because the concepts would become embedded in basic medical training, is a longer-term prospect that must be preceded by first upskilling the teachers. Academics, together with the broader range of disaster healthcare professionals, and ideally nonclinical emergency managers, therefore comprise the target audience for initial DEH education and their needs can be most readily met by postgraduate-level short courses.

These courses can be offered on-line to reach an international community and accelerate the take up and further development of DEH. They may also be certificated and, where appropriate, assigned credit for study towards more extensive, degree-bearing awards. In addition, short courses introducing simple but effective DEH applications can be designed to increase the disaster preparedness of the general public - an increasingly important issue since it is evident that modern ICTs, especially mobile technologies, empower ordinary citizens to be active responders, not just passive victims, in disaster situations.

Recalling that the DEH project is a work in progress, the education and training theme therefore attempts to advance the role of DEH in DMED practice by designing and offering a short, on-line course on the subject. The next section considers the course objectives and some design considerations, and illustrates these factors by recommending topics and an overall structure.

\section{DEH Curriculum: Objectives, Design Considerations, and Course Topics}

The main DEH course objectives are to present the concepts of eHealth, demonstrate the potential value of DEH in disaster situations, and ensure students understand the utility of the tools needed to introduce and integrate DEH into their own practice. These three goals - concepts, value, and utility - comprise a triad designed to impart knowledge and competencies in the use of DEH and the ability to follow or construct applicable protocols for disaster situations. Evidently, course 
design has to be collaborative involving DMED, DMAN, and eHealth experts and what follows is intended as a starting point for discussion and planning.

An opening topic for the DEH course is terminology. The WADEM initiative recognised concerns over some of the definitions used in DMED; adding meanings from the DEH domain has the potential to compound confusion. Students will be made aware of these issues and provided with a short taxonomy that will identify and resolve possible misunderstandings.

To achieve the design goals, a primary requirement is to acquaint students with key eHealth technologies such as the electronic health record (EHR), telehealth, decision support systems, RFID, data analytics, etc, as well as ICT application areas such as mobile technologies, cloud computing, social media etc. ${ }^{11}$ The curriculum will provide examples of the use of these technologies and devices in both normal and disaster situations emphasising data acquisition, analysis, and information sharing rather than hardware. ${ }^{30}$ The approach also needs to be holistic, recognising that information and possible eHealth solutions must be shared horizontally as well as vertically, and also appropriately with all personnel engaged in the emergency setting, not just DMED specialists. These personnel include emergency managers (making a connection with the inter-agency communication theme of the DEH project) and ordinary citizens and volunteers.

Case studies will help students to assess the potential of eHealth applications judged, for example, on the basis of care quality, access to care, and cost. They will also learn how to evaluate scenarios and redesign applications to improve the efficacy and efficiency of care. Students will be made aware of the limitations of technologies, eg, the difficulty of making electronic triage assessments when there is no electrical power, or the problem of returning a disaster setting to normal operating conditions (eg merging temporary and normal electronic health records).

The course will offer students experience in creating eHealth competencies and protocols, which are a standard feature of DEM practice.

It will also study the role of DEH throughout the disaster cycle demonstrating contributions that $\mathrm{DEH}$ could make in disaster mitigation, and preparedness (eg competency and protocol design), as well as in response and recovery. A good example of the value of DEH in the recovery phase is the use of telehealth to assist patients suffering from long-term mental illnesses incurred by their disaster experiences. ${ }^{31}$ Unobtrusive measuring devices are available to monitor these patients in their own homes where they are most comfortable, and to alert carers to the need for intervention.

Time will be devoted to the role of epidemiology in disasters and the contribution that big data techniques and data visualisation software advances will make to epidemiological approaches to disaster planning and response. ${ }^{32}$

Independently, disaster situations and eHealth applications raise their own questions of ethical practice, privacy, confidentiality, safety, and legality. ${ }^{33}$ The course will cover relevant aspects referring to any differences of approach and responsibility that distinguish disaster situations from normal circumstances.

An aspect of disaster management and healthcare that is often overlooked is the way in which a crisis changes leadership roles. ${ }^{34}$ A DMAN manager and a DMED specialist may have clear authority in their separate domains but in a disaster, when victims have to be evacuated or health priorities decided, the requirement for cross-agency team work can raise contradictions and territorial conflicts. The curriculum will raise students' awareness of the potential for disagreements that could compromise care and familiarise them with negotiating techniques and how to establish protocols, to resolve, or better, avoid these issues.

The topics described in the above paragraphs reveal several of the key subject areas that would be expected in a short course on disaster eHealth for healthcare practitioners. The list is not intended to be comprehensive but to present a coherent picture of DEH and its potential, and highlight aspects that can be used to enhance awareness and facilitate adoption. The topics and discussion therefore offer a starting point for a formal design process (see later) that could lead to a course structure along the following lines.

1. Course overview and terminology of disaster management, medicine, and eHealth

2. Current practice in disaster management

3. Current practice in disaster medicine

4. Current practice in eHealth 
5. Disaster eHealth applications throughout the disaster cycle

6. Disaster eHealth scenarios: design, selection, and evaluation

7. Disaster eHealth competencies, protocols, and policy

8. Disaster risk assessment, safety, and security

9. Public health law and ethics

10. Leadership and communication

\section{Future Developments}

The development of a strategy for disaster eHealth education and training that is the subject of this paper is an important part of an on-going project to characterise DEH, discover its benefits and limitations, and promote its adoption by the disaster community wherever it can improve disaster healthcare.

The education project is at an early stage with a pilot course based on the model described in this paper is currently under development at the University of Nebraska and there are many design and operational issues to address. Foremost amongst these is the formation of a panel of experts who will design the proposed DEH short course and its operation. Key issues include, naturally, the course topics and progression, the role of theory and practical elements, the delivery mode, perhaps as a MOOC with an organisation such as the Khan Academy, ${ }^{35}$ or EDX, ${ }^{36}$ and the recognition of student success.

There is also the need to ensure that students are exposed to research methods and innovation in related fields. Fee structures, university participation, interaction with the health and disaster management sectors and with international organisations, are other factors important for raising awareness of DEH and encouraging sponsorship.

These developments, both in the short- and longer-term futures, will enhance the scope and scale of disaster eHealthcare by saving lives and reducing the health impacts of disasters.

\section{Corresponding author:}

\section{Tony Norris}

School of Engineering, Computer and Mathematical Sciences Auckland University of Technology Auckland
New Zealand

E-mail:tonorris@aut.ac.nz

Web: http://www.aut.ac.nz/profiles/tony-norris

Conflict of Interest. The authors declare no conflicts of interest.

\section{Funding acknowledgement:}

The authors gratefully acknowledge the award of grants to the DEH project from the Royal Society of New Zealand, Auckland University of Technology, the University of Agder, and the University of Nebraska, Omaha

\section{References}

1. Al-Shaqsi S, Gauld R, Lovell S, et al. Challenges of the New Zealand healthcare disaster preparedness prior to the Canterbury earthquakes: A qualitative analysis. N Z Med J (Online) 2013;126(1371):9-18.

2. Centre for Research on the Epidemiology of Natural Disasters (CRED). (2015). The human cost of natural disasters. Université Catholique de Louvain. Available at: http://emdat.be/ accessed 15 December 2016.

3. Warfield, C. (2008). The disaster management cycle. Available at:

http://www.gdrc.org/uem/disasters/1$\mathrm{dm}$ cycle.html accessed 20 March 2018.

4. Hogan DE, Burstein JL. Disaster medicine. Second edition, Philadelphia: Lippincott, Williams and Wilkins, 2007.

5. Subbarao I, Lyznicki JM, Hsu B, et al. A consensus-based educational framework and competency set for the discipline of disaster medicine and public health preparedness. Disaster Med Public Health Prep 2008;2(1):57-68.

6. Galea S. The long-term health consequences of disasters and mass traumas. Can Med Assoc J 2007; 176(9):1293-1294.

7. Hristidis, V, Chen S-C, Li T, Luis S, Deng Y. Survey of data management and analysis in disaster situations. J Syst Softw 2010;83:17011714.

8. National Academies Press. Improving disaster management: The role of IT in mitigation in preparedness, response, and recovery. Washington DC: National Academies Press, 2007. 
9. James JJ, Walsh L. E-health in preparedness and response. Disaster Med Public Health Prep 2011;5(4):257-258.

10. Sakanushi K, Hieda T, Shiraishi T, et al,. Electronic triage system for continuously monitoring casualties at disaster scenes. $J$ Ambient Intell Human Comput 2013;4:547-558.

11. Coiera E. Guide to Health Informatics. Third edition, Boca Raton: CRC Press, 2015.

12. Norris AC, Martinez S, Labaka L, et al.. Disaster e-health: A new paradigm for collaborative healthcare in disasters. Proceedings of the $12^{\text {th }}$ International Conference on Information Systems for Crisis Response and Management (ISCRAM) 2015

13. Russo C. (2011) Emergency communication remains a challenge ten years after $9 / / 11$. Homeland Security News Wire. Available at: http://www.homelandsecuritynewswire.com/emer gency-communication-remains-challenge-tenyears-after-911 accessed 20 March 2018.

14. Bissell RA. Public health and medicine in emergency management. In: McEntire DA, editor. Disciplines, disasters, and emergency management, Emmitsburg: FEMA; 2005.

15. Abbas R, Norris AC, Parry DT. Pinpointing what is wrong with cross-agency collaboration in disaster healthcare. $J$ Int Soc Telemed eHealth 2018;6:e3

16. Association of American Medical Colleges (AAMC). Training future physicians on weapons of mass destruction: Report of the expert panel on bioterrorism education for medical students, Washington DC: AAMC, 2003.

17. World Association for Disaster and Emergency Medicine (WADEM). (2016). Available at: https://wadem.org/ accessed 15 December 2016.

18. Seynaeve G, Archer F, Fisher J, et al. International standards and guidelines on education and training for the multi-disciplinary health response to major events that threaten the health status of a community. Prehosp Disaster Med 2004;19(2):S17-30.

19. Scott LA, Carson DS, Greenwell IB. Disaster 101: A novel approach to disaster medicine training for health professionals. $J$ Emerg Med 2010;39(2);220-226.

20. Pfenninger EG, Domres, BD, Stahl W, et al. Medical student disaster medicine education: The development of an educational resource. Int $J$ Emerg Med 2010;3:9-20.

21. Della Corte F, Hubloue I, Gallardo AR, et al. The European Masters degree in disaster medicine (EMDM): A decade of exposure. Front Public Health 2014;2:1-3.

22. Smith J, Levy MJ, Hsu EB, Levy JL. Disaster curricula in medical education: Pilot survey. Prehosp Disaster Med 2012;27(5):492-494.

23. Mortelmans LCJ, Lievers J, Dieltiens G, Sabbe, MB. Are Belgian military students in medical sciences better educated in disaster medicine than their civilian colleagues? Prehosp Disaster Med 2016; 162:383386.

24. Ragazonni L, Ingrassia PL, Gugliotta G, et al. Italian medical students and disaster medicine: Awareness and formative needs. Amer J Disaster Medicine 2013;8 (2):127-136.

25. Franc JM, Nichols D, Dong SL. Increasing emergency medicine residents' confidence in disaster management: Use of an emergency department simulator and an expedited curriculum. Prehosp Disaster Med 2012;27(1):31-35.

26. Ingrassia PL, Foletti M, Djalali A, et al. Education and training initiatives for crisis management in the European Union: A web-based analysis of available programs. Prehosp Disaster Med 2014;29(2):115-126.

27. Ingrassia PL, Ragazzoni L, Tengattini M, Carenzo L, Della Corte F. Nationwide program of education for undergraduates in the field of disaster medicine: Development of a core curriculum centered on blended learning and simulation Tools. Prehosp Disaster Med 2014;29(5):1-8.

28. Collaborating Centre for Oxford University and CUHK for Disaster and Medical Humanitarian Response (CCOUC). (2015) Free online course: Public health principles in disaster and medical humanitarian response. Available at: https://phpidccouc.conted.ox.ac.uk/ accessed 15 December 2016.

29. Emergency Medicine Cape Town (EMCT). (2016) Disaster medicine. Available at: http://www.emct.info/disaster-medicine.html accessed 15 December 2016.

30. Mutasa M. Investigating the significance of disaster information management. J Disaster Risk Stud 2013;5(2):1-6. 
31. Augusterfer EF, Mollica RF, Lavelle J. A review of telemental health in international and postdisaster settings. Int Rev Psychiatry 2015;27:540546.

32. Malilay J, Heumann M, Perrotta D, et al. The role of applied epidemiology methods in the disaster management cycle. Am J Public Health 2014;104:2092-2102.

33. Call JA, Pfefferbaum B, Jenuwine MJ, Flynn BW. Practical legal and ethical considerations for the provision of acute disaster mental health services. Psychiatry 2012;75(4):305-322.

34. Demiroz F, Kapucu N. The role of leadership in managing emergencies and disasters. Europ $J$ Econ Pol Stud 2012;5(1):91-101.

35. Khan Academy. (2016) Available at: https://www.khanacademy.org/ accessed 15 December 2016.

36. EDX. (2016) Available at:

https://www.edx.org/course accessed 15 December 2016. 